\title{
Análises químicas da matéria orgânica presente em amostras lunares
}

\author{
Zita Martins \\ Centro de Química-Física Molecular - Institute of Nanoscience and Nanotechnology (CQFM-IN), and Institute for Bioengineering and Biosciences \\ (iBB), Departamento de Engenharia Química, Instituto Superior Técnico (IST), Universidade de Lisboa, Portugal \\ zita.martins@tecnico.ulisboa.pt
}

\begin{abstract}
Chemical analysis of organic matter present in lunar samples - Primitive celestial bodies, particularly those containing ice, preserve information that goes back to the formation of the solar system. Hence, research on primitive celestial bodies is crucial to explain the origin and evolution of these bodies and the origin of life in our solar system. The permanently shaded regions (PSRs) of the Moon's polar ice preserve volatile and organic compounds that have been delivered by comets and/or asteroids. On the other hand, the lunar regolith contains organic compounds, but its origin was recently determined to be terrestrial contamination during the handling of samples returned by the Apollo space missions in the 1970s. The chemical analyses of the organic matter present in lunar samples shows the importance of the curation of primitive materials from our solar system.
\end{abstract}

Corpons Corpos celestes primitivos, em particular aqueles que contên gelo, preservam informações que renontam à formação do sistema solar. Por isso, a pesquisa sobre corpos celestes primitivos é crucial para explicar a origem e evolução desses corpos e a origem da vida no nosso sistema solar. As regiões permanentemente sombreadas (PSRs) dos gelos polares da Lua preservam compostos voláteis e orgânicos que foram entregues por cometas e/ou asteroides. Por outro lado, o rególito lunar contém compostos orgânicos, mas a sua origem foi recentemente determinada como sendo contaminação terrestre durante o manuseamento das amostras das missões espaciais Apollo na década de 1970. As análises químicas da matéria orgânica presentes em amostra lunares mostram a importância da curadoria na preservação de materiais primitivos do nosso sistema solar.

\section{Química do gelo lunar polar}

A evolução química e a origem da vida no sistema solar são eventos que permanecem sem explicação. Informação sobre as condições físicas e químicas durante a formação do nosso sistema solar pode ser obtida estudando corpos primitivos, em particular corpos ricos em gelo que preservam informações inalteradas desde o início do sistema solar. Dados obtidos por missões espaciais e/ou através de análise de amostras de corpos celestes gelados fornecem clarificação sobre os processos químicos que modificaram os materiais originais do sistema solar, bem como sobre a síntese dos blocos moleculares de construção da vida e seus precursores. O gelo está presente em muitos locais do sistema solar, incluindo as regiões permanentemente sombreadas (permanently shadowed regions - PSRs) dos gelos lunares polares, que funcionam como uma cápsula do tempo. O Satélite de Observação e Deteção de Crateras Lunares (Lunar Crater Observation and Sensing Satellite - LCROSS) detetou um reservatório de gelos nas crateras da região polar sul da Lua [1-3]. As moléculas (e abundâncias relativas) detetadas por refletância no infravermelho próximo no material ejetado pelo impacto da missão espacial LCROSS no polo sul lunar estão apresentadas na Tabela 1 [1]. Mais detalhes sobre estes resultados estão descritos no artigo deste número especial [3].

As regiões permanentemente sombreadas dos gelos lunares polares receberam, e subsequentemente preservaram, compostos voláteis e compostos orgânicos que foram entregues ao sistema Terra-Lua primordial por cometas e/ ou asteroides (Figura 1, [4,5]). O gelo lunar presente em crateras de impacto [2] também preserva informação sobre moléculas orgânicas complexas formadas aquando do impacto de cometas [6]. Por exemplo, a energia gerada pelo impacto de cometas e meteoritos em superfícies geladas produz vários aminoácidos (Figura 2, [6]). Desta forma, as crateras de impacto representam um testemunho do passado geológico e químico da superfície da Lua [2]. Tudo isto fornece um cenário único em termos de preservação da história de compostos voláteis e moléculas orgânicas na Lua, um registro que não é preservado em outras partes do sistema solar interno. A análise in situ de amostras de PSRs na Lua nunca foi realizada, e poderá de futuro revelar as reações fotoquímicas que ocorreram em gelos interplanetários antes de sua captura nos polos lunares (imitando a química da superfície em gelos interestelares) [6-8].

Tabela 1 - Abundâncias relativas dos compostos detetados no material ejetado pelo impacto da missão espacial LCROSS na cratera Cabeus do polo sul lunar (através de espectroscopia de refletância no infravermelho próximo) [1].

\begin{tabular}{|c|c|}
\hline Composto & Abundância relativa à água $\mathrm{H}_{2} \mathrm{O}(\mathrm{g})(\mathrm{em} \%)$ \\
\hline $\mathrm{H}_{2} \mathrm{O}$ & 100 \\
\hline $\mathrm{H}_{2} \mathrm{~S}$ & 16.75 \\
\hline $\mathrm{NH}_{3}$ & 6.03 \\
\hline $\mathrm{SO}_{2}$ & 3.19 \\
\hline $\mathrm{C}_{2} \mathrm{H}_{4}$ & 3.12 \\
\hline $\mathrm{CO}_{2}$ & 2.17 \\
\hline $\mathrm{CH}_{3} \mathrm{OH}$ & 1.55 \\
\hline $\mathrm{CH}_{4}$ & 0.66 \\
\hline $\mathrm{OH}$ & 0.03 \\
\hline
\end{tabular}




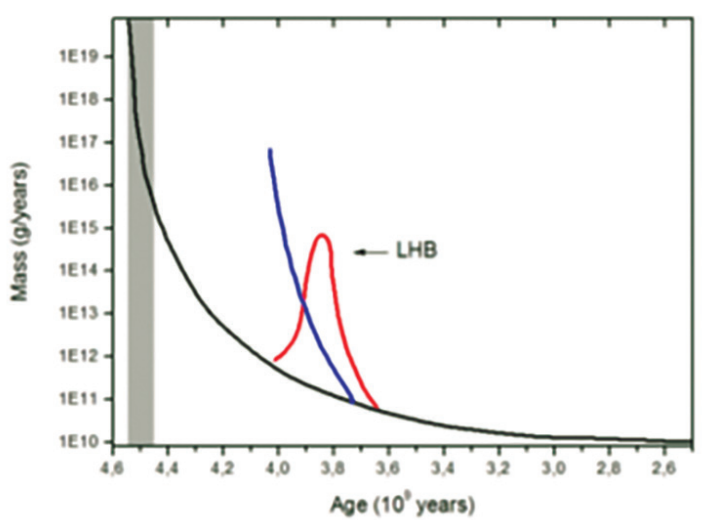

Figura 1 - Estimativas da taxa de acreção de impactos na superfície da Lua (de [4]; adaptado de [5]). A linha preta é uma extrapolação do atual fluxo restringido com dados de impacto lunar. A linha vermelha mostra o evento de Bombardeamento Tardio Pesado ("Late Heavy Bombardment" - LHB) da datação de bacias de impacto lunar e modelação da dinâmica do sistema solar primitivo. A linha azul mostra uma curva de acreção muito improvável.

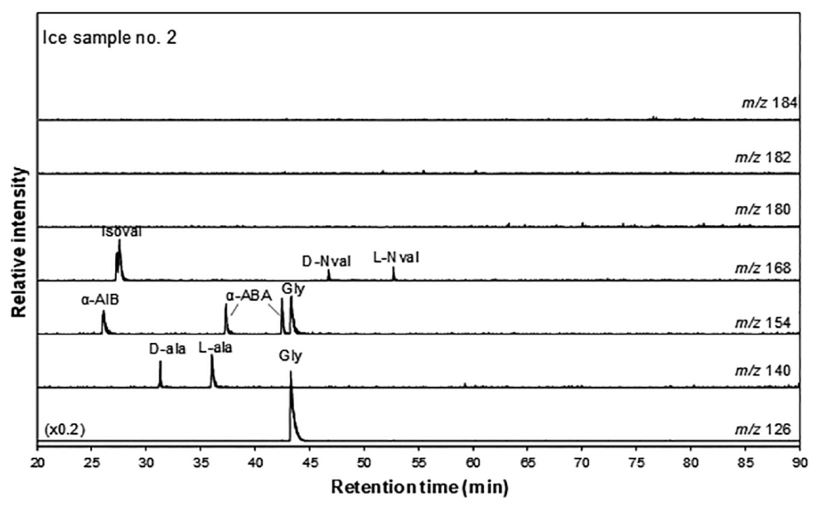

Figura 2 - O impacto de um cometa numa superfície planetária gera energia suficiente para sintetizar compostos orgânicos. Por exemplo, foi mostrado experimentalmente que o impacto de uma mistura gelada de composição análoga a um cometa produz vários aminoácidos, incluindo $\alpha$-aminoácidos, como mostrado nos cromatogramas ( $\mathrm{m} / \mathrm{z} 126,140,154$, 168, 180, 182 and 184) da cromatografia gasosa acoplada a espectrometria de massa [6]. A mistura gelada análoga a um cometa que deu origem aos aminoácidos continha $\mathrm{NH}_{4} \mathrm{OH}$ (28\% em solução de amoníaco gasoso dissolvido em $\mathrm{H}_{2} \mathrm{O}$ ), $\mathrm{CO}_{2}$ e $\mathrm{CH}_{3} \mathrm{OH}$ na relação molar de 9,1:8:1, tendo o impacto uma velocidade de $7 \mathrm{~km} / \mathrm{s}$. A identificação dos aminoácidos derivatizados ( $N$-TFA, $O$-isopropil) é a seguinte: Gly = glicina; D-ala = D-alanina; L-ala = L-alanina; $\alpha$-AIB = ácido $\alpha$-aminoisobutírico; $\alpha$-ABA = ácido $\alpha$-aminobutírico; Isoval = D,L-isovalina; D-Nval = D-norvalina; L-Nval = L-norvalina.

\section{Compostos orgânicos em rególito lunar}

Para além da análise do gelo lunar, também o rególito lunar tem sido alvo de análises de compostos químicos desde as missões Apollo nos anos 1970 até aos dias de hoje. Pequenas quantidades de hidrocarbonetos, HCN, DCN e aminoácidos foram detetados no rególito lunar [9-17]. Até aos recentes estudos de Elsila e colegas [18], a origem exata das moléculas orgânicas detetadas em amostras lunares permaneceu desconhecida, tendo sido sugerido quatro potenciais origens: i) contaminação terrestre durante o manuseamento e análise das amostras lunares, ii) contaminação terrestre a partir do motor de escape do módulo lunar, iii) vento solar, e iv) entrega exógena por meteoritos carbonáceos, cometas ou partículas de poeira interplanetária [10,12,14,17,19-22]. As limitações do equipamento analítico usado no século passado (e.g. limites de deteção elevados, impossibilidade de separar enantiómeros, e ausência de equipamento para realizar análises isotópicas de compostos específicos) levaram a que não fosse possível fazer um estudo preciso sobre compostos orgânicos indígenas do rególito lunar.

Em 2016 Elsila e colegas [18] detetaram glicina, D- e L-alanina, D- e L-ácido aspártico, D- e L-ácido glutâmico, De L-serina, L-treonina, L-valina, ácido $\alpha$-aminoisobutírico, D- e L-ácido $\beta$-aminobutírico, ácido $\alpha$-aminobutírico, ácido $\gamma$-aminobutírico, $\beta$-alanina, e ácido $\varepsilon$-aminocaproico em amostras do rególito lunar das missões Apollo 16 e Apollo 17 que tinham sido armazenadas pela NASA, utilizando cromatografia líquida de ultra-alta eficiência com deteção de fluorescência e espetrometria de massa de tempo de voo. Os aminoácidos estavam presentes em baixas concentrações (de 0,2 partes por mil milhões (ppb) a 651,1 ppb), e a maioria dos aminoácidos proteinogénicos apresentavam um excesso do enantiómero L, indicando uma origem terrestre. Estes investigadores realizaram também análises isotópicas de compostos específicos de carbono da glicina, da $\beta$-alanina (ácido 3-aminopropanoico) e da L-alanina na amostra de rególito Apollo 70011, tendo obtido os valores de $\delta^{13} \mathrm{C}-21 \%$ a $-33 \%$, o que é consistente com valores terrestres de aminoácidos (Figura 3, [18]). De facto, estes valores de $\delta^{13} \mathrm{C}$ não se enquadram na gama de valores do vento solar ou de meteoritos carbonáceos (Figura 3). Para determinar a origem exata da contaminação terrestre (i.e., manuseamento das amostras lunares e/ou motor de escape do módulo lunar), a equipa comparou a abundância total de aminoácidos de uma amostra de rególito lunar recolhida debaixo do módulo lunar e outra recolhida a $6,5 \mathrm{~km}$ de distância. Se o motor de escape do módulo lunar fosse uma fonte de contaminação, a concentração total de aminoácidos deveria ser muito mais elevada do que na outra amostra recolhida a $6,5 \mathrm{~km}$. A equipa não observou diferenças na concentração total de aminoácidos entre a amostra de rególito lunar recolhida debaixo do módulo lunar e outra recolhida a $6,5 \mathrm{~km}$ de distância, claramente indicando que a origem terrestre dos aminoácidos detetados no rególito lunar deverá ter ocorrido durante o processo de manuseamento das amostras (durante os anos de 1970).

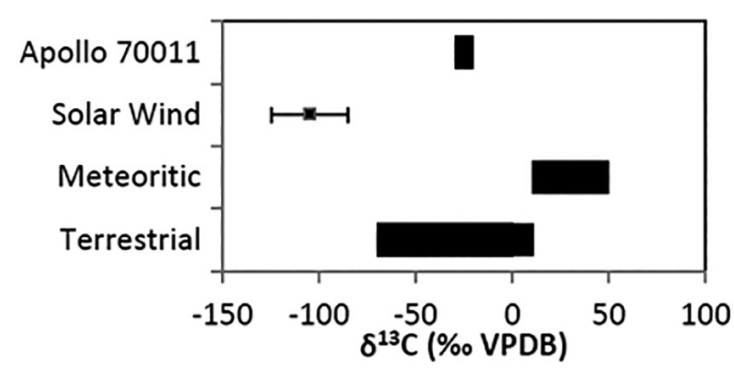

Figura 3 - Gama de valores de $\delta^{13} \mathrm{C}$ medidos para aminoácidos na amostra de rególito lunar Apollo 70011, e comparação com os valores para o vento solar, meteoritos carbonáceos, e fontes biológicas terrestres [18].

As análises de compostos orgânicos em rególito lunar mostram claramente a importância da curadoria na preservação de amostras de corpos celestes, e as implicações para futuras missões espaciais (in situ e/ou de retorno de amostras para a Terra) às regiões permanentemente som- 
breadas dos gelos lunares polares. Presentemente várias agências espaciais confirmam a importância da curadoria, investindo bastantes recursos humanos e equipamento para preservar amostras extraterrestres, e preparando a receção de amostras de futuras missões espaciais que irão certamente contribuir para desvendar a origem da vida na Terra e possivelmente noutras partes do nosso sistema solar.

\section{Referências}

[1] A. Colaprete, P. Schultz, J. Heldmann, D. Wooden, M. Shirley, K. Ennico, B. Hermalyn, W. Marshall, A. Ricco, R. C. Elphic, D. Goldstein, D. Summy, G. D. Bart, E. Asphaug, D. Korycansky, D. Landis, L. Sollitt, Science 330 (2010) 463-468.

[2] P. Dias, P. Pina, Química - Bol. SPQ 43 (2019) 113-118.

[3] P.M. Machado, Química - Bol. SPQ 43 (2019) 105-108.

[4] H. Cottin, J.M. Kotler, K. Bartik, H.J. Cleaves, C.S. Cockell, J.-P.P. de Vera, P. Ehrenfreund, S. Leuko, I.L. ten Kate, Z. Martins, R. Pascal, R. Quinn, P. Rettberg, F. Westall, Space Sci. Rev. 209 (2017) 1-42.

[5] C. Koeberl, Geol. Soc. Am. Spec. Pap. 405 (2006) 1-22.

[6] Z. Martins, M.C. Price, N. Goldman, M.A. Sephton, M.J. Burchell, Nature GeoScience 6 (2013) 1045-1049.

[7] M.P. Bernstein, J.P. Dworkin, S.A. Sandford, G.W. Cooper, L.J. Allamandola, Nature 416 (2002) 401-403.

[8] G.M.M. Caro, U.J. Meierhenrich, W.A. Schutte, B. Barbier, A.A. Segovia, H. Rosenbauer, W.H.-P. Thiemann, A. Brack, J.M. Greenberg, Nature 416 (2002) 403-406.

[9] P.E. Hare, K. Harada, S.W. Fox, Geochim. Cosmochim. Acta 2 (1970) 1799-1803.

[10] K. Harada, P.E. Hare, C.R. Windsor, S.W. Fox, Science 173 (1971) 433-435.
[11] B. Nagy, C.M. Drew, P.B. Hamilton, V.E. Modzeles, M.E. Murphy, W.M. Scott, H.C. Urey, M. Young, Science 167 (1970) 770-773.

[12] B. Nagy, J.E. Modzeleski, V.E. Modzeleski, M.A.J. Mohammad, L.A. Nagy, W.M. Scott, C.M. Drew, J.E. Thomas, R. Ward, P.B. Hamilton, H.C. Urey, Nature 232 (1971) 94-98.

[13] C.W. Gehrke, R.W. Zumwalt, K. Kuo, J.J. Rash, W.A. Aue, D.L. Stalling, K.A. Kvenvolden, C. Ponnamperuma, Space Life Sci. 3 (1972) 439-449.

[14] S.W. Fox, K. Harada, P.E. Hare, Proc. Fourth Lunar Sci. Conf. Geochim. Cosmochim. Acta 2 (1973) 2241-2248.

[15] V.E. Modzeleski, J.E. Modzeleski, M.A. Mohammed, L.A. Nagy, B. Nagy, W.S. McEwan, H.C. Urey, P.B. Hamilton, Nat. Phys. Sci. 242 (1973) 50-52.

[16] P.T. Holland, B.R. Simoneit, P.C. Wszolek, A.L. Burlingame, Proc. Lunar Sci. Conf. 2 (1972) 2131-2147.

[17] K.L.F. Brinton, J.L. Bada, Geochim. Cosmochim. Acta 60 (1996) 349-354.

[18] J.E. Elsila, M.P. Callahan, J.P. Dworkin, D.P. Glavin, H.L. McLain, S.K. Noble, E.K. Gibson Jr. Geochim. Cosmochim. Acta 172 (2016) 357-369.

[19] D.A. Flory, B.R. Simoneit, Space Life Sci. 3 (1972) 457-468.

[20] S.W. Fox, K. Harada, P.E. Hare, Geochim. Cosmochim. Acta 40 (1976) 1069-1071.

[21] C.W. Gehrke, R.W. Zumwalt, K. Kuo, C. Ponnamperuma, A. Shimoyama, Orig. Life Evol. Biosphere 6 (1975) 541-550.

[22] K.L. Thomas-Keprta, S.J. Clemett, S. Messenger, D.K. Ross, L. Le, Z. Rahman, D.S. McKay, E.K. Gibson Jr, C. Gonzalez, W. Peabody, Geochim. Cosmochim. Acta 134 (2014), 1-15.

Atualidades CientíFicas

\section{Síntese eficiente de benzofuranos e benzotiofenos}

Estruturas contendo benzofuranos e benzotiofenos acilados em C3, e seus derivados, podem ser encontradas em muitos produtos naturais e compostos biologicamente ativos. Os métodos tradicionais para a obtenção destes compostos envolvem principalmente a reação de cicloadição de derivados fenólicos com 1,3-dicarbonilos, a condensação de acetofenonas substituídas com N,N-dimetilformamida dimetilacetal, bem como a acilação C3 direta de benzofuranos e benzotiofenos. No entanto, a maioria destes métodos exige a presença de uma quantidade estequiométrica de ácidos de Lewis, não raras vezes com utilização limitada no que respeita aos substratos usados. Portanto, têm sido desenvolvidas estratégias novas e eficientes para a síntese destes compostos.

Investigadores da Universidade de Jiangnan, China, usaram uma estratégia de transferência de átomo de hidrogénio (HAT) catalisada por ferro, para desenvolver uma reação de ciclização eficiente de 1,6-eninos para obter 3-acilbenzo-

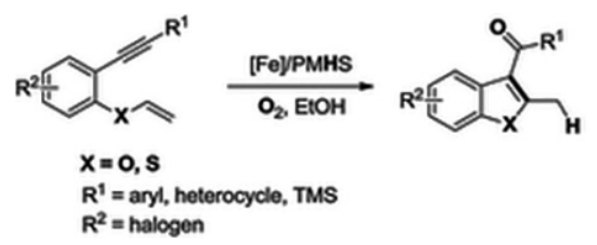

Fonte: furanos e 3-acilbenzotiofenos. Foi proposta e confirmada uma sequência reacional envolvendo uma transferência de átomo de hidrogénio/ciclização e inserção de $\mathrm{O}_{2}$. Os investigadores descrevem o seu método sintético como sendo viável para a obtenção de valiosos compostos heteroaromáticos, experimentalmente simples e seguro e que utiliza reagentes baratos e amigos do ambiente.

Hydrofunctionalization of 1,6-Enynes,https://www.chemistryviews.org/details/news/11121041/Hydrofunctionalization_ of_16-Enynes.html (Acedido em 28/12/2018).

X.-F. Xia, W. He, G.-W. Zhang, D. Wanga, Org. Chem. Front. 6 (2019) 342-346. 